\title{
A New Sliding Mode Control for Satellite Formation Flying in Elliptical Orbits Based on a Model in the True Anomaly Region
}

\author{
By Zhen Chen, ${ }^{1,2)}$ Xin $L v,{ }^{1,2)}$ XiangDong LiU, ${ }^{1,2)}$ Binglong Cong ${ }^{1,2)}$ and Yafei $\mathrm{LI}^{1,2)}$ \\ ${ }^{1)}$ School of Automation, Beijing Institute of Technology, Beijing, China \\ ${ }^{2)}$ Key Laboratory for Intelligent Control \& Decision of Complex Systems, Beijing Institute of Technology, Beijing, China
}

(Received July 11th, 2013)

\begin{abstract}
In order to achieve precise maneuvers of formation flying satellites, an accurate and controllable relative motion model is greatly needed. However, most transformation equations only take either external disturbances or $J_{2}$ perturbation into consideration, which will affect satellites' long-term reconfiguration. Therefore, nonlinear relative motion equations of satellites in elliptic orbits with consideration of both external disturbances and $J_{2}$ perturbation are derived in this paper. To obtain accurate formations and improve the system robustness in the presence of external disturbances and $J_{2}$ perturbation, a reconfiguration and formation-keeping control law based on the nonlinear sliding mode function is proposed, where nonlinear gains of exponential growth are used to replace the constant gain of the traditional sliding mode function. The proposed control law restrains the control law saturation during big errors and improves the response rate during small errors, and effectively enhances the rate of reaching the sliding mode surface. Finally, the numerical simulation results verify the effectiveness of the proposed strategies.
\end{abstract}

Key Words: Relative Motion, $J_{2}$ Perturbations, Bounded External Disturbances, Lyapunov Transformation, Nonlinear SM Function

\author{
Nomenclature \\ a: semi-major axis \\ $e$ : eccentricity \\ $i$ : inclination \\ $\Omega$ : right ascension \\ $\omega$ : argument of perigee \\ $f$ : true anomaly \\ Subscripts \\ D: Deputy \\ $C$ : Chief
}

\section{Introduction}

Satellite formation flying (SFF) is revolutionizing our way of performing space-based operations, and this new paradigm brings several advantages for space missions such as simpler design, lower fabrication cost and faster launches, but the main advantage lies in reliability and flexibility compared with a single, complex vehicle. A common way to implement the SFF is the leader/follower approach. In this method, the object of the control is to adjust the follower's positions which are relative to the leader and drive it to track the desired relative trajectory. Several research studies have been done to implement SFF. ${ }^{1,2)}$ However, flying autonomously in a precise formation is still a challenge. ${ }^{3)}$ Thus an accurate and controllable relative motion model and elaborate control of each spacecraft in formation are greatly needed. In recent years, several studies have been made related to the dynamics and control of multiple satellites flying

(C) 2014 The Japan Society for Aeronautical and Space Sciences in formation.

To provide optimal and robust control to satellite formation is highly dependent on detailed mathematical models of formation flying. The simplest model to describe the relative motion between two satellites is known as Hill's equation. ${ }^{4)}$ However, Hill's equations are based on the assumptions that the reference orbit is circular, the Earth is spherically symmetric, and the chief orbit is very close to the reference orbit such that there are no external disturbances and the nonlinear terms in the relative motion can be neglected. These assumptions will lead to unacceptable errors in the long-term prediction of the relative motion for SFF. Thus an extension to elliptic Keplerian orbits is proposed, which is known as Tschauner-Hempel equations, ${ }^{5)}$ yet still assuming no orbital perturbations. With the need for highly precise formations arising, a more detailed model for satellites was greatly needed, especially taking $J_{2}$ perturbation into account. As a result, Wong et al. ${ }^{6)}$ proposed full nonlinear dynamics to replace the Hill linearization, which included full nonlinear dynamics and disturbances. Later in Manikonda et al. ${ }^{7)}$ and Yan et al., methods were derived for arbitrary orbital eccentricity and with added terms for orbital perturbations. Another method to describe the relative motion of satellites is to use orbit elements, ${ }^{8)}$ in accordance with Soop's theory. In current studies, most models in elliptic orbits presented only take one kind of disturbance (external disturbances or $J_{2}$ perturbations) into consideration, ignoring the combined effects of both to the SFF. That may lead to the imprecision of control and the waste of fuel. Thus a more accurate solution for the relative motion is needed. In this paper, a nonlinear relative motion equation including both external 
disturbances and $J_{2}$ perturbations is presented. As more complex models of SFF appear, highly precise and robust controllers there will be demanded.

Sliding mode control (SMC) is a nonlinear robust control method, which is widely applied in SFF control for its strong robustness against disturbances and uncertainties. Yeh et al. ${ }^{9)}$ developed an effective robust method control strategy for satellites using SMC based on Hill's equations. That method determined parameters such as the equivalent damping ratio, bandwidth and thrust, so that fuel cost was minimized. Hui and $\mathrm{Li}^{10)}$ proposed a terminal SMC for spacecraft formation flying based on nonlinear relative dynamics of circular reference orbit. Pongvthithum et al. ${ }^{11)}$ presented an universal adaptive control of satellite formation flying based on elliptic reference including external disturbances. The idea of control design is to combine the domination design and the monotone adaptive gain, which guarantees position tracking and full adaptivity against all parameters.

The SMC system has the property of being sensitive to uncertainties and disturbances, while this characteristic is ensured only after the system states reach the sliding surface, and the robustness is not guaranteed during the reaching phase. Considering the proportionality relationship of the reaching phase and initial value of the sliding mode function, reducing the initial value of the sliding mode function is an effective way to enhance system robustness. In large-scale reconfiguration tasks, the existing constant gain SM may not guarantee fast convergence rate and may also induce control saturation and overconsumption of fuel. In many SFF applications, the thrust level is very low and the initial offset may generally take many hours and even days to settle. Hence, for a high-precision SFF reconfiguration control with strict arrival schedules, the existing SMC may have restrictions. Additionally, most of the presented SMCs for spacecraft formation control are about circular reference orbits or elliptic orbits while ignoring the effect of $J_{2}$ perturbations on the orbits element.

In this paper, sliding mode controllers based on the nonlinear SM function are designed for SFF in elliptical reference orbits subject to external disturbances in the presence of $J_{2}$ perturbations and without $J_{2}$ perturbations, which use nonlinear gains of exponential growth to replace the constant gain of the traditional SM function. That is, for large errors, the control effort is reduced, and the initial value of the SM function is decreased simultaneously, consequently getting rid of the problem of saturation induced by the large errors and shortening the reaching phase in the meantime. Otherwise, the control effort is increased exponentially in the neighborhood of the reference operating point, which ensures fast convergence. That solves the problem of large errors leading to saturation, small errors causing slow response, which also improving the robustness of the system. Therefore, in the proposed controller design, system states move on the sliding surface faster and the fuel cost for the initial offset is also lower than the traditional SMC. It is assumed that satellites move in low Earth orbits and two kinds of relative motion models are proposed: one takes $J_{2}$ perturbations into consideration; one does not. In order to facilitate the design of the control law, the relative motion is linearized by feedback linearization.

This paper is organized as follows. Section 1 is the introduction. Section 2 introduces the equations of the relative motion based on perturbation and without consideration of $J_{2}$ perturbation, and meanwhile, the equations are linearized by feedback linearization. Section 3 formulates the SMC base nonlinear SM function for the linearized equations. Section 4 presents the simulation results. Section 5 presents the conclusion.

\section{Equations for Relative Motion}

\subsection{Relative motion equation based on $\boldsymbol{J}_{2}$ perturbation}

The relative motion of two satellites in elliptical orbits is depicted in Fig. 1. The satellite which flies on the reference orbit is labeled as the chief satellite and the other as the deputy satellite. Let $B_{I}=\left\{i_{x}, i_{y}, i_{z}\right\}$ be the inertial coordinate system, which is a non-rotating frame with its origin at the center of the Earth. Here $i_{x}$ and $i_{z}$ are aligned with the equinox and spin axis of the Earth, respectively. The coordinate system $B_{L}=\left\{i_{r}, i_{\theta}, i_{h}\right\}$ is attached to the moving frame of the chief spacecraft with $i_{r}$ lying along the radius vector from the Earth's center to the chief satellite, and $i_{h}$ along the normal vector to the orbital plane of the chief satellite, where $i_{\theta}=i_{h} \times i_{r}$. In Fig. $1, r_{C}$ and $r_{D}$ are the position vectors of the chief satellite and the deputy satellite. To describe the formation configuration geometrically, the formation vector $l$ is proposed, with $l=l_{x} i_{r}+l_{y} i_{\theta}+l_{z} i_{h}{ }^{12)}$ Define $r_{T}$ to be the expected position vector of the chaser satellite, so $r_{T}=r_{C}+l$. From Fig. 1 it is easy to obtain $\rho=$ $r_{D}-r_{C}=x i_{r}+y i_{\theta}+z i_{h}$ and $\Delta \rho=r_{D}-r_{T}=\Delta x i_{r}+$ $\Delta y i_{\theta}+\Delta z i_{h}$. Let $\delta=[a, e, i, \Omega, \omega, f]^{\mathrm{T}}$ be the vector of orbit elements of the chief. As the influence of $J_{2}$ perturbations, the orbit elements are changing with time. From Ref. 13) the total angular velocity for osculating elements including the effect of $J_{2}$ is

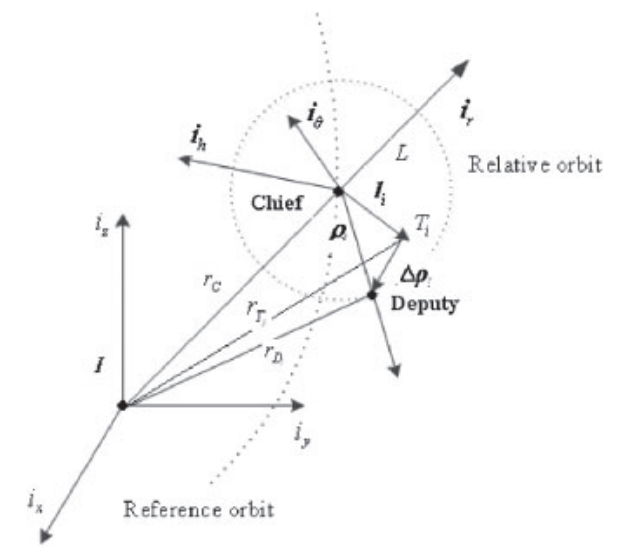

Fig. 1. Illustration of a chief-deputy type of a two-spacecraft formation. 


$$
\begin{aligned}
& \varpi_{x}=\dot{\Omega} \sin \theta \sin i+\dot{i} \cos \theta \\
& \varpi_{y}=0 \\
& \varpi_{z}=\dot{f}+\dot{\omega}+\dot{\Omega} \cos i
\end{aligned}
$$

where $\theta=f+\omega$ represents the true argument of latitude. The time variations of the orbital elements caused by $J_{2}$ are obtained from Lagrange's planetary equations. ${ }^{14)}$

$$
\begin{aligned}
\dot{\Omega} & =-\frac{3 J_{2} R_{e q}^{2}}{a^{3.5} \eta^{7}} \sqrt{\mu}(1+e \cos f)^{3} \cos i \sin ^{2} \theta \\
\dot{i} & =-\frac{3 J_{2} R_{e q}^{2}}{4 a^{3.5} \eta^{7}} \sqrt{\mu}(1+e \cos f)^{3} \sin 2 i \sin 2 \theta \\
\dot{\theta} & =n \frac{\sqrt{\mu}(1+e \cos f)^{2}}{\eta^{3}}
\end{aligned}
$$

$$
+\frac{3 J_{2} R_{e q}^{2}}{a^{3.5} \eta^{7}} \sqrt{\mu}(1+e \cos f)^{3} \cos ^{2} i \sin ^{2} \theta
$$

where $n=\sqrt{\mu / a^{3}}$ and $\eta^{2}=1-e^{2}$, and the time rate of the true anomaly is

$$
\begin{aligned}
\dot{f}= & \frac{\sqrt{\mu}(1+e \cos f)^{2}}{a^{1.5} \eta^{3}}+\frac{\alpha}{2} \frac{\sqrt{\mu}(1+e \cos f)}{e a^{3.5} \eta^{7}} \\
& \times\left[\left(3 \sin ^{2} i \sin ^{2} \theta-1\right)(1+e \cos f) \cos f\right. \\
& \left.+(2+e \cos f) \sin f \sin ^{2} i \sin 2 \theta\right]
\end{aligned}
$$

The relative dynamics of the deputy in the presence of $J_{2}$ perturbations and bounded external disturbances with respect to the moving frame $B_{L}$ can be written as

$$
\begin{aligned}
{\left[\begin{array}{c}
\ddot{x} \\
\ddot{y} \\
\ddot{z}
\end{array}\right]=} & -2\left[\begin{array}{ccc}
0 & -\varpi_{z} & \varpi_{y} \\
\varpi_{z} & 0 & -\varpi_{x} \\
-\varpi_{y} & \varpi_{x} & 0
\end{array}\right]\left[\begin{array}{c}
\dot{x} \\
\dot{y} \\
\dot{z}
\end{array}\right]-\left[\begin{array}{ccc}
0 & -\dot{\varpi}_{z} & \dot{\varpi}_{y} \\
\dot{\varpi}_{z} & 0 & -\dot{\varpi}_{x} \\
-\dot{\varpi}_{y} & \dot{\varpi}_{x} & 0
\end{array}\right]\left[\begin{array}{l}
x \\
y \\
z
\end{array}\right] \\
& -\left[\begin{array}{ccc}
0 & -\varpi_{z} & \varpi_{y} \\
\varpi_{z} & 0 & -\varpi_{x} \\
-\varpi_{y} & \varpi_{x} & 0
\end{array}\right]\left[\begin{array}{ccc}
0 & -\varpi_{z} & \varpi_{y} \\
\varpi_{z} & 0 & -\varpi_{x} \\
-\varpi_{y} & \varpi_{x} & 0
\end{array}\right]\left[\begin{array}{l}
x \\
y \\
z
\end{array}\right] \\
+ & {\left[\begin{array}{c}
-\frac{\mu}{r_{D}^{3}}\left(x+r_{C}\right)+\frac{\mu}{r_{C}^{2}} \\
-\frac{\mu}{r_{F}^{3}} y \\
-\frac{\mu}{r_{F}^{3}} z
\end{array}\right]+a_{F J 2}-a_{C J 2}+u_{D}+d }
\end{aligned}
$$

where $\mu$ is the gravitational parameter, and $u_{D}=\left[u_{D}^{x}, u_{D}^{y}, u_{D}^{z}\right]$ are the control accelerations. Define

$$
\begin{aligned}
& u_{G}=\left(\frac{1+e \cos f}{a\left(1-e^{2}\right)}\right)^{3}\left[\begin{array}{c}
2 \mu \Delta x \\
-\mu \Delta y \\
-\mu \Delta z
\end{array}\right] \\
& u_{D}^{g x} \triangleq \frac{\mu}{r_{D}^{3}}\left(x+r_{C}\right)-\frac{\mu}{r_{C}^{2}} \\
& u_{D}^{g y} \triangleq \frac{\mu}{r_{D}^{3}} y \\
& u_{D}^{g z} \triangleq \frac{\mu}{r_{D}^{3}} z \\
& u_{l}=-2\left[\begin{array}{ccc}
0 & -\varpi_{z} & \varpi_{y} \\
\varpi_{z} & 0 & -\varpi_{x} \\
-\varpi_{y} & \varpi_{x} & 0
\end{array}\right]\left[\begin{array}{l}
i_{x} \\
i_{y} \\
\dot{l}_{z}
\end{array}\right]-\left[\begin{array}{ccc}
0 & -\dot{\varpi}_{z} & \dot{\varpi}_{y} \\
\dot{\varpi}_{z} & 0 & -\dot{\varpi}_{x} \\
-\dot{\varpi}_{y} & \dot{\varpi}_{x} & 0
\end{array}\right]\left[\begin{array}{l}
l_{x} \\
l_{y} \\
l_{z}
\end{array}\right] \\
& -\left[\begin{array}{ccc}
0 & -\varpi_{x} & \varpi_{y} \\
\varpi_{z} & 0 & -\varpi_{x} \\
-\varpi_{y} & \varpi_{x} & 0
\end{array}\right]\left[\begin{array}{ccc}
0 & -\varpi_{x} & \varpi_{y} \\
\varpi_{z} & 0 & -\varpi_{x} \\
-\varpi_{y} & \varpi_{x} & 0
\end{array}\right]\left[\begin{array}{l}
l_{x} \\
l_{y} \\
l_{z}
\end{array}\right]-\left[\begin{array}{l}
\ddot{l}_{x} \\
\ddot{l}_{y} \\
\ddot{l}_{z}
\end{array}\right] \\
& u_{1}=u_{D}-u_{g}-u_{G}-u_{l}
\end{aligned}
$$




$$
\begin{aligned}
\Delta(X, t)= & -2\left[\begin{array}{ccc}
0 & -\varpi_{z} & \varpi_{y} \\
\varpi_{z} & 0 & -\varpi_{x} \\
-\varpi_{y} & \varpi_{x} & 0
\end{array}\right]\left[\begin{array}{l}
\dot{x} \\
\dot{y} \\
\dot{z}
\end{array}\right]-\left[\begin{array}{ccc}
0 & -\dot{\varpi}_{z} & \dot{\varpi}_{y} \\
\dot{\varpi}_{z} & 0 & -\dot{\varpi}_{x} \\
-\dot{\varpi}_{y} & \dot{\varpi}_{x} & 0
\end{array}\right]\left[\begin{array}{l}
x \\
y \\
z
\end{array}\right] \\
& -\left[\begin{array}{cccc}
0 & -\varpi_{z} & \varpi_{y} \\
\varpi_{z} & 0 & -\varpi_{x} \\
-\varpi_{y} & \varpi_{x} & 0
\end{array}\right]\left[\begin{array}{ccc}
0 & -\varpi_{z} & \varpi_{y} \\
\varpi_{z} & 0 & -\varpi_{x} \\
-\varpi_{y} & \varpi_{x} & 0
\end{array}\right]\left[\begin{array}{l}
x \\
y \\
z
\end{array}\right] \\
& +a_{D J_{2}}-a_{C J_{2}}-\left[\begin{array}{c}
2 \dot{f} \Delta \dot{y}+\ddot{f} \Delta y+\dot{f}^{2} \Delta x \\
-2 \dot{f} \Delta \dot{x}-\ddot{f} \Delta x+\dot{f}^{2} \Delta y \\
0
\end{array}\right]
\end{aligned}
$$

where $a_{D J_{2}}$ and $a_{C J_{2}}$ denote $J_{2}$ gravitational perturbations per unit mass of deputy and chief described in terms of the local vertical, local horizontal (LVLH) of the chief, respectively. $\left[\begin{array}{lll}x_{I D} & y_{I D} & z_{I D}\end{array}\right]$ and $\left[\begin{array}{lll}x_{I C} & y_{I C} & z_{I C}\end{array}\right]$ are position vectors of the deputy and chief represented in the inertial coordinate system.

$a_{D J_{2}}=\left(-\frac{3}{2}\right) J_{2}\left(\frac{\mu}{r_{D}^{2}}\right)\left(\frac{R_{e q}}{r_{D}}\right)^{2} R_{I L}\left[\begin{array}{l}\left(1-5\left(\frac{z_{I D}}{r_{D}}\right)^{2}\right) \frac{x_{I D}}{r_{D}} \\ \left(1-5\left(\frac{z_{I D}}{r_{D}}\right)^{2}\right) \frac{y_{I D}}{r_{D}} \\ \left(3-5\left(\frac{z_{I D}}{r_{D}}\right)^{2}\right) \frac{z_{I D}}{r_{D}}\end{array}\right]$

$a_{C J_{2}}=\left(-\frac{3}{2}\right) J_{2}\left(\frac{\mu}{r_{C}^{2}}\right)\left(\frac{R_{e q}}{r_{C}}\right)^{2} R_{I L}\left[\begin{array}{l}\left(1-5\left(\frac{z_{I C}}{r_{C}}\right)^{2}\right) \frac{x_{I C}}{r_{C}} \\ \left(1-5\left(\frac{z_{I C}}{r_{C}}\right)^{2}\right) \frac{y_{I C}}{r_{C}} \\ \left(3-5\left(\frac{z_{I C}}{r_{C}}\right)^{2}\right) \frac{z_{I C}}{r_{C}}\end{array}\right]$

where $J_{2}$ is the second zonal harmonic coefficient of the Earth, $R_{e q}$ is the Earth's equatorial radius, and $R_{I L}$ is the rotation matrix of the inertial coordinate to the chief's LVLH. $^{15)}$

$$
R_{I L}=R_{z}(\theta) R_{x}(i) R_{z}(\Omega)
$$

where $R_{x}(\cdot), R_{y}(\cdot)$ and $R_{z}(\cdot)$ are the rotation matrices which rotate around the $x$-axis, $y$-axis and $z$-axis, respectively.

$$
R_{x}(\delta)=\left[\begin{array}{ccc}
1 & 0 & 0 \\
0 & \cos \delta & \sin \delta \\
0 & -\sin \delta & \cos \delta
\end{array}\right]
$$

$$
\begin{aligned}
& R_{y}(\delta)=\left[\begin{array}{ccc}
\cos \delta & 0 & -\sin \delta \\
0 & 1 & 0 \\
\sin \delta & 0 & \cos \delta
\end{array}\right] \\
& R_{z}(\delta)=\left[\begin{array}{ccc}
\cos \delta & \sin \delta & 0 \\
-\sin \delta & \cos \delta & 0 \\
0 & 0 & 1
\end{array}\right]
\end{aligned}
$$

Then, Eq. (4) can be rewritten by feedback linearization $^{16)}$ as

$$
\left[\begin{array}{c}
\Delta \ddot{x} \\
\Delta \ddot{y} \\
\Delta \ddot{z}
\end{array}\right]=\left[\begin{array}{c}
2 \dot{f} \Delta \dot{y}+\ddot{f} \Delta y+\dot{f}^{2} \Delta x \\
-2 \dot{f} \Delta \dot{x}-\ddot{f} \Delta x+\dot{f}^{2} \Delta y \\
0
\end{array}\right]+u_{1}+d+\Delta(X, t)
$$

Considering the design need in the following, we introduce other imaginary orbit elements $a_{1}, e_{1}, i_{1}, \Omega_{1}, \omega_{1}$ and $f_{1}$. The initial values of these orbit elements are the same as the chief's, and are not affected by $J_{2}$ perturbations. Now assume true anomaly $f_{1}$ as a free variable, and the Lyapunov transformation ${ }^{17,18)}$ is introduced.

$$
\begin{aligned}
& \Delta x_{1}=\frac{1+e_{1} \cos f_{1}}{p_{0}} \Delta x \\
& \Delta y_{1}=\frac{1+e_{1} \cos f_{1}}{p_{0}} \Delta y \\
& \Delta z_{1}=\frac{1+e_{1} \cos f_{1}}{p_{0}} \Delta z
\end{aligned}
$$

Then Eq. (12) becomes rather simpler in dimensionless form as follows.

$$
\begin{aligned}
{\left[\begin{array}{c}
\Delta x_{1}^{\prime \prime} \\
\Delta y_{1}^{\prime \prime} \\
\Delta z_{1}^{\prime \prime}
\end{array}\right]=} & {\left[\begin{array}{c}
\frac{3}{1+e_{1} \cos f_{1}} \Delta x_{1}+2 \Delta y_{1}^{\prime} \\
-2 \Delta x_{1}^{\prime} \\
-\Delta z_{1} \\
\left(1-e_{1}^{2}\right)^{3}
\end{array}\right] } \\
& +\frac{\left(1+e_{1} \cos f_{1}\right)^{3} n_{1}^{2}}{p_{0}(1}
\end{aligned}
$$




$$
\begin{aligned}
& +\frac{\left(1-e_{1}^{2}\right)^{3}}{p_{0}\left(1+e_{1} \cos f_{1}\right)^{3} n_{1}^{2}} d \\
& +\frac{\left(1-e_{1}^{2}\right)^{3}}{p_{0}\left(1+e_{1} \cos f_{1}\right)^{3} n_{1}^{2}} \Delta(X, t)
\end{aligned}
$$

where ()$=\mathrm{d}() / \mathrm{d} f_{1}, p_{0}$ is a constant. Then, rewrite Eq. (14) in matrix form as

$X_{1}^{\prime}\left(f_{1}\right)=A_{1}\left(f_{1}\right) X_{1}\left(f_{1}\right)+B_{1}\left(f_{1}\right) u_{1}+B_{1} d+B_{1} \Delta(X, t)$

where

$$
A_{1}\left(f_{1}\right)=\left[\begin{array}{cccccc}
0 & 0 & 0 & 1 & 0 & 0 \\
0 & 0 & 0 & 0 & 1 & 0 \\
0 & 0 & 0 & 0 & 0 & 1 \\
\frac{3}{1+e_{1} \cos f_{1}} & 0 & 0 & 0 & 2 & 0 \\
0 & 0 & 0 & -2 & 0 & 0 \\
0 & 0 & -1 & 0 & 0 & 0
\end{array}\right]
$$

$$
B_{1}\left(f_{1}\right)=\frac{\left(1-e_{1}^{2}\right)^{3}}{p_{0}\left(1+e_{1} \cos f_{1}\right)^{3} n^{2}}\left[\begin{array}{ccc}
0 & 0 & 0 \\
0 & 0 & 0 \\
0 & 0 & 0 \\
1 & 0 & 0 \\
0 & 1 & 0 \\
0 & 0 & 1
\end{array}\right]
$$

Assume that the true anomaly of the target satellite is $f_{*}$ at time $t_{*}$. Define

$$
\begin{aligned}
& \begin{array}{r}
X\left(t_{*}\right)=\left[\Delta x\left(t_{*}\right) \Delta y\left(t_{*}\right) \Delta z\left(t_{*}\right) \Delta \dot{x}\left(t_{*}\right) \Delta \dot{y}\left(t_{*}\right) \Delta \dot{z}\left(t_{*}\right)\right]^{\mathrm{T}} \\
X_{2}\left(f_{*}\right)=\left[\Delta x\left(f_{*}\right) \Delta y\left(f_{*}\right) \Delta z\left(f_{*}\right) \Delta x^{\prime}\left(f_{*}\right) \Delta y^{\prime}\left(f_{*}\right) \Delta z^{\prime}\left(f_{*}\right)\right.
\end{array} \\
& X_{1}\left(f_{*}\right)=\left[\Delta x_{1}\left(f_{*}\right) \Delta y_{1}\left(f_{*}\right) \Delta z_{1}\left(f_{*}\right) \Delta x_{1}^{\prime}\left(f_{*}\right) \Delta y_{1}^{\prime}\left(f_{*}\right) \Delta z^{\prime}\right. \\
& \text { thus } \begin{array}{r}
X_{2}\left(f_{*}\right)=P_{1}\left(f_{*}\right) X\left(t_{*}\right) \\
X_{1}\left(f_{*}\right)=P_{2}\left(f_{*}\right) X_{2}\left(f_{*}\right)
\end{array}
\end{aligned}
$$$$
X_{2}\left(f_{*}\right)=\left[\Delta x\left(f_{*}\right) \Delta y\left(f_{*}\right) \Delta z\left(f_{*}\right) \Delta x^{\prime}\left(f_{*}\right) \Delta y^{\prime}\left(f_{*}\right) \Delta z^{\prime}\left(f_{*}\right)\right]^{\mathrm{T}}
$$$$
X_{1}\left(f_{*}\right)=\left[\Delta x_{1}\left(f_{*}\right) \Delta y_{1}\left(f_{*}\right) \Delta z_{1}\left(f_{*}\right) \Delta x_{1}^{\prime}\left(f_{*}\right) \Delta y_{1}^{\prime}\left(f_{*}\right) \Delta z_{1}^{\prime}\left(f_{*}\right)\right]^{\mathrm{T}}
$$

where

$$
\begin{aligned}
& P_{1}\left(f_{*}\right)=\left[\begin{array}{cccccc}
1 & 0 & 0 & 0 & 0 & 0 \\
0 & 1 & 0 & 0 & 0 & 0 \\
0 & 0 & 1 & 0 & 0 & 0 \\
0 & 0 & 0 & \dot{f}_{*}^{-1} & 0 & 0 \\
0 & 0 & 0 & 0 & \dot{f}_{*}^{-1} & 0 \\
0 & 0 & 0 & 0 & 0 & \dot{f}_{*}^{-1}
\end{array}\right]
\end{aligned}
$$

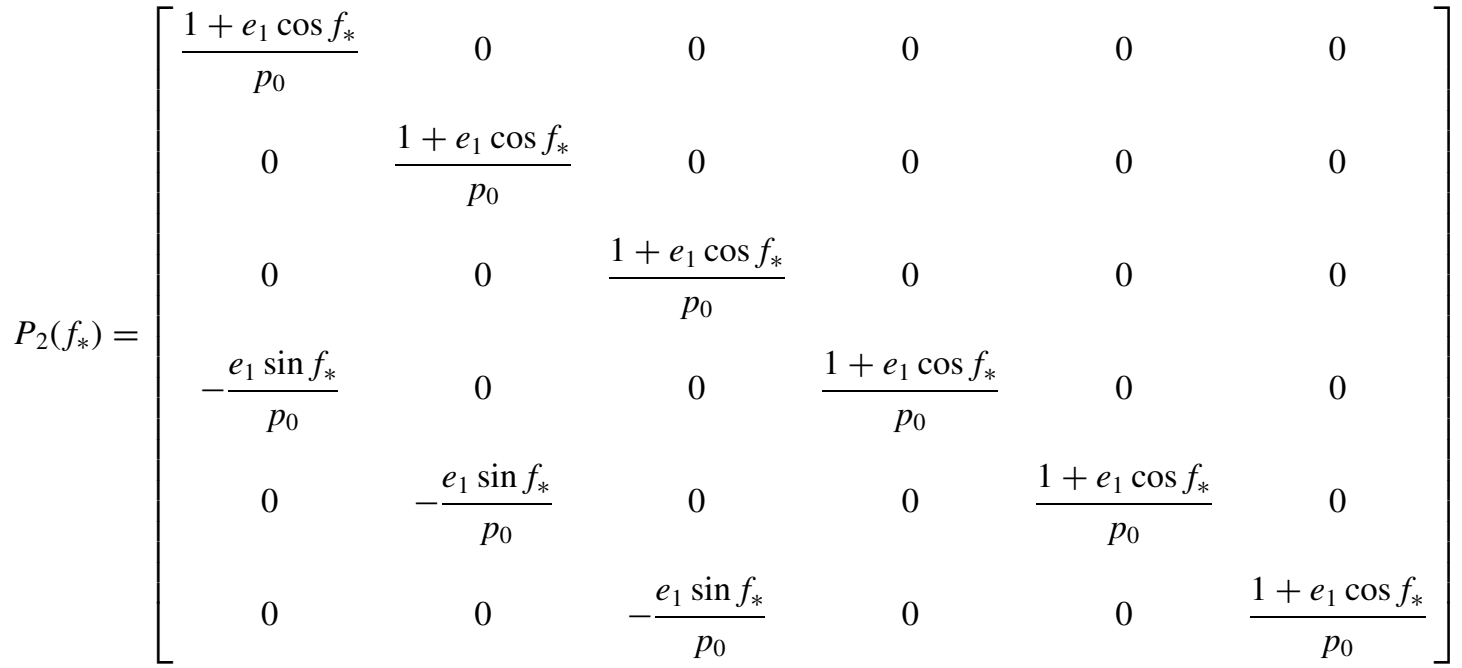

In order to facility the control design in the following, one set is

$$
\begin{aligned}
q_{1} & =\left[\begin{array}{lll}
\Delta x_{1}(t) & \Delta y_{1}(t) & \Delta z_{1}(t)
\end{array}\right]^{\mathrm{T}} \\
q_{2} & =\left[\begin{array}{lll}
\Delta x_{1}^{\prime}(t) & \Delta y_{1}^{\prime}(t) & \Delta z_{1}^{\prime}(t)
\end{array}\right]^{\mathrm{T}} \\
v & =\frac{\left(1-e_{1}^{2}\right)^{3}}{p_{0}\left(1+e_{1} \cos f_{1}\right)^{3} n_{1}^{2}} u_{1}
\end{aligned}
$$

$$
d_{1}=\frac{\left(1-e_{1}^{2}\right)^{3}}{p_{0}\left(1+e_{1} \cos f_{1}\right)^{3} n_{1}^{2}} d
$$

Thus the state equations can be changed into $q_{1}^{\prime}=q_{2}$ $q_{2}^{\prime}=A_{11}\left(f_{1}\right) q_{1}+A_{12}\left(f_{1}\right) q_{2}+B v+B d_{1}+B \Delta_{1}(X, t)$ 
where

$$
\begin{aligned}
A_{11} & =\left[\begin{array}{ccc}
\frac{3}{1+\cos f_{1}} & 0 & 0 \\
0 & 0 & 0 \\
0 & 0 & -1
\end{array}\right], A_{12}=\left[\begin{array}{ccc}
0 & 2 & 0 \\
-2 & 0 & 0 \\
0 & 0 & 0
\end{array}\right], \\
B & =\left[\begin{array}{lll}
1 & 0 & 0 \\
0 & 1 & 0 \\
0 & 0 & 1
\end{array}\right]
\end{aligned}
$$

2.2. Relative motion equation without $J_{2}$ perturbations If the equation of SFF does not take $J_{2}$ perturbations into consideration, the variables $\Omega, \omega, i$ in Eq. (1) are constants, so their derivation are zeros. Then relative dynamics of the deputy and chief without $J_{2}$ perturbations can be written as

$$
\begin{aligned}
& \ddot{x}=2 \dot{f} y+\ddot{f} y+\dot{f}^{2} x-\frac{\mu}{r_{D}^{3}}\left(x+r_{C}\right)+\frac{\mu}{r_{C}^{2}}+u_{D}^{x}+d_{x} \\
& \ddot{y}=-2 \dot{f} x-\ddot{f} x+\dot{f}^{2} y-\frac{\mu}{r_{D}^{3}} y+u_{D}^{y}+d_{y} \\
& \ddot{z}=-\frac{\mu}{r_{D}^{3}} z+u_{D}^{z}+d_{z}
\end{aligned}
$$

where

$$
\begin{aligned}
& \dot{f}=\sqrt{\frac{\mu}{r_{C}^{3}}(1+e \cos f)} \\
& \ddot{f}=-2 e \frac{\mu}{r_{C}^{3}} \sin f
\end{aligned}
$$

Then, the free variable time $t$ is replaced by true anomaly $f$ in the same way as in the last section. Thus Eq. (18) can be changed into

$$
X_{1}^{\prime}(f)=A_{1}(f) X_{1}(f)+B_{1}(f) u_{1}+B_{1} d
$$

where $A_{1}(f), B_{1}(f)$ have the same expression with Eq. (15), and the state in $f$ and in $t$ has the same relationship with Eq. (16).

Similarly, the state equations without $J_{2}$ perturbations can be changed into

$$
\begin{aligned}
& q_{1}^{\prime}=q_{2} \\
& q_{2}^{\prime}=A_{11}(f) q_{1}+A_{12}(f) q_{2}+B v+B d_{1}
\end{aligned}
$$

where the expressions of $A_{11}(f), A_{12}(f), B$ are the same as Eq. (17).

The objective of this paper is to compute the thrust required to drive the deputy satellite to a desired formation trajectory, with respect to the chief satellite, with bounded external disturbances under the existence of $J_{2}$ perturbations or without $J_{2}$ perturbations with low fuel consumption and good robustness.

Remark 1: $J_{2}$ perturbations and external disturbances are bounded by

$$
\left\|d_{1}\right\| \leq d_{\max } \quad\left\|\Delta_{1}(X, t)\right\| \leq k_{J_{2}} J_{2}\left\|X_{2}\right\|
$$

where $d_{\max }$ and $k_{J_{2}}$ are positive constants.

\section{Controller Design Base Nonlinear SM Function}

Generally, traditional SMC is defined as

$$
s=q_{2}+c q_{1}
$$

where $s=\left[\begin{array}{lll}s_{1} & s_{2} & s_{3}\end{array}\right]^{\mathrm{T}}$ and $c$ are sliding surfaces and slopes of sliding surfaces, respectively.

The $f_{1}$ derivative of $s$ is

$$
s^{\prime}=q_{2}^{\prime}+c q_{2}
$$

Theorem 1: For the system characterized by Eq. (17), by choosing the SMC law with the form of Eq. (21)

$$
\begin{aligned}
v= & -A_{11}\left(f_{1}\right) q_{1}-A_{12}\left(f_{1}\right) q_{2}-c q_{2} \\
& -\left(\alpha+k J_{2}\left\|X_{2}\right\|\right) \operatorname{sgn}(s)
\end{aligned}
$$

The closed-loop system is globally asymptotically stable if the condition $\alpha>d_{\max }$ is satisfied.

Proof: Define the following positive definite Lyapunov function:

$$
V=\frac{1}{2} s^{\mathrm{T}} s
$$

The $f_{1}$ derivative of $V$ is given by

$$
V^{\prime}=s^{\mathrm{T}} s^{\prime}
$$

Substituting Eq. (22) and Eq. (17) into Eq. (25), by some simplifications, the $f_{1}$ derivative of the Lyapunov function can be given as follows

$$
\begin{aligned}
V^{\prime} & =-(\alpha-d)\|s\|-\left(k J_{2}\left\|X_{2}\right\|-\Delta_{1}(X, t)\right)\|s\| \\
& \leq-\left(\alpha-d_{\max }\right)\|s\|-\left(k J_{2}\left\|X_{2}\right\|-\Delta_{1}(X, t)\right)\|s\|
\end{aligned}
$$

As $\alpha>d_{\max }$, then $V^{\prime}<0$ for any $q_{1}, q_{2}$. According to the Lyapunov stability principle, the conclusion that the system is asymptotically stable can be drawn.

While considering the characteristics of SMC, the gain $c$ proportionally affects the sliding mode control law. If too large a $c$ is chosen, when the deputy satellite is far away from the desired position, the actuator will generate a large control law to force the deputy satellite to the desired position. This may lead to actuator saturation at the initial time. In addition, too large a $c$ can increase the initial value of the SM function, and this will lead to a reduced rate of reaching the sliding mode surface. Contrarily, if too small a $c$ is chosen, the control effort decreases exponentially in the neighborhood of the reference operation point, and a direct consequence of this is a drop in system response rate.

Remark 2: $c$ is the key factor to determine the amplitude of the initial control value and the system response rate. At the condition of the same initial errors, if too small a gain $c$ is chosen, $s\left(t_{0}\right)$ will decrease, and then the reaching time will be reduced at the same time; the initial value of the control law will decrease, or otherwise.

Aiming to solve this problem, we propose a SM controller based on the nonlinear SM function, which solves the problem of "large error making saturation, small error causing 
slow response." The design concept of this SMC is to replace the constant gain by nonlinear exponential gain. That is, the control effort is reduced when the states are far away from the reference operating point; on the other hand, for small errors the controller ensures a fast convergence rate.

The nonlinear SM function is described as follows.

$$
s=q_{2}+k_{c} e^{-k_{1}\left|S\left(q_{1}\right)\right|} q_{1}
$$

In the case of large-scale maneuvering, it may lead the nonlinear part to converge to zero, and then to disable the control actuator. The hyperbolic tangent function is adopted as the exponential power.

$$
S\left(q_{1}\right)=\tanh \left(q_{1}\right)
$$

The characteristics of the hyperbolic tangent function are:

1) When the independent variable is zero, the function value is zero

2) It is strictly increasing and its differential is continuous

3 ) It is a bounded function and its limit value is \pm 1 .

Therefore, when the error $q_{1}$ is large enough, one has the following relationship

$$
-k_{1}\left|S\left(q_{1}\right)\right| \approx-k_{1}
$$

Thus one can choose suitable gain $k_{1}$ according to different needs; when the error $q_{1}$ is relative small, one has

$$
k_{1}\left|S\left(q_{1}\right)\right|>k_{1} q_{1} \rightarrow 0
$$

Obviously, for the time being, the system response is faster than the sliding mode control base constant gain.

The derivative of $s$ with respect to $f_{1}$ is

$s^{\prime}=q_{2}^{\prime}+k_{c} e^{-k_{1}\left|\tanh \left(q_{1}\right)\right|} q_{2}-k_{c} e^{-k_{1}\left|\tanh \left(q_{1}\right)\right|} \frac{k_{1}}{\cosh ^{2}\left(q_{1}\right)} q_{2}\left|q_{1}\right|$

Theorem 2: For the system characterized by Eq. (16), by choosing the nonlinear SM function with the form of Eq. (27), and control law determined by Eq. (30),

$$
v=v_{*}+v_{1}
$$

where

$$
\begin{aligned}
v_{*}= & -A_{11}\left(f_{1}\right) q_{1}-A_{12}\left(f_{1}\right) q_{2}-k_{c} e^{-k_{1}\left|\tanh \left(q_{1}\right)\right|} q_{2} \\
& +k_{c} k_{1} e^{-k_{1}\left|\tanh \left(q_{1}\right)\right|} / \cosh ^{2}\left(q_{1}\right)\left|q_{1}\right| q_{2} \\
v_{1}= & -\left(\alpha+k J_{2}\left\|X_{2}\right\|\right) \operatorname{sgn}(s) \quad \alpha>d_{\max }
\end{aligned}
$$

the tracking errors can converge to zero under the condition of both $J_{2}$ perturbations and bounded external disturbances.

Proof: Define the following positive definite Lyapunov function.

$$
V=\frac{1}{2} s^{\mathrm{T}} s
$$

The true anomaly $f_{1}$ derivative of $V$ is

$$
V^{\prime}=s^{\mathrm{T}} s^{\prime}
$$

Substituting Eq. (17) and Eq. (29) into Eq. (32), we have

$$
\begin{aligned}
V^{\prime}= & s^{\mathrm{T}} s^{\prime} \\
= & s^{\mathrm{T}}\left(q_{2}^{\prime}+k_{c} e^{-k_{1}\left|\tanh \left(q_{1}\right)\right|} q_{2}+k_{c} e^{-k_{1}\left|\tanh \left(q_{1}\right)\right|} q_{2}\right. \\
& \left.-k_{c} e^{-k_{1}\left|\tanh \left(q_{1}\right)\right|} \frac{k_{1}}{\cosh ^{2}\left(q_{1}\right)} q_{2}\left|q_{1}\right|\right) \\
= & s^{\mathrm{T}}\left(A_{11}(f) q_{1}+A_{12}(f) q_{2}+B_{1} v+B_{1} d+B_{1} \Delta_{1}(X, t)\right. \\
& \left.-k_{c} e^{-k_{1}\left|\tanh \left(q_{1}\right)\right|} q_{2}+k_{c} e^{-k_{1}\left|\tanh \left(q_{1}\right)\right|} \frac{k_{1}}{\cosh ^{2}\left(q_{1}\right)} q_{2}\left|q_{1}\right|\right)
\end{aligned}
$$

Combining with Eq. (30), we obtain

$$
\begin{aligned}
V^{\prime} & =-(\alpha-d)\|s\|-\left(k J_{2}\left\|X_{2}\right\|-\Delta_{1}(X, t)\right)\|s\| \\
& \leq-\left(\alpha-d_{\max }\right)\|s\|-\left(k J_{2}\left\|X_{2}\right\|-\Delta_{1}(X, t)\right)\|s\|
\end{aligned}
$$

Considering $\alpha>d_{\max }, k J_{2}\left\|X_{2}\right\| \geq\left\|\Delta_{1}(X, t)\right\|$. So $V^{\prime}<0$ for any $q_{1}, q_{2}$. Therefore, according to the Lyapunov stability criterion, it can be proved that $q_{1}, q_{2}$ converge to the vicinity of the origin, locally and asymptotically. Moreover, if $\|s\| \rightarrow \infty$, then $V \rightarrow \infty$ can be easily obtained, which shows that the closed-loop system is globally asymptotically stable. That is to say, the satellites can form the desired geometry formation with the control law designed in this paper. The proof is completed.

Theorem 3: For the system characterized by Eq. (20), we choose the nonlinear SM function with the form of Eq. (27), and control law determined by Eq. (35). If the condition $\alpha>$ $d_{\max }$ is satisfied, the closed-loop system is globally asymptotically stable. Thus

$$
v=v_{*}+v_{1}
$$

where

$$
\begin{aligned}
v_{*}= & -A_{11}(f) q_{1}-A_{12}(f) q_{2}-k_{c} e^{-k_{1}\left|\tanh \left(q_{1}\right)\right|} q_{2} \\
& +k_{c} k_{1} e^{-k_{1}\left|\tanh \left(q_{1}\right)\right|} / \cosh ^{2}\left(q_{1}\right)\left|q_{1}\right| q_{2} \\
v_{1}= & -\alpha \operatorname{sgn}(s) \quad \alpha>d_{\max }
\end{aligned}
$$

Proof: The process of proof is the same as the proof in theorem 2. For simplification, the certification is ignored.

Remark 3: From the characteristic of the nonlinear SM function, one can see, at initial time, the initial value of the nonlinear SM function is relatively small as the effect of exponent gain with comparison to the traditional SM function, so the time of reaching the sliding mode surface is shortened and the initial fuel consumption is decreased. As a result, the robustness of the system is improved and as the system errors converge to zero, the nonlinear SM gain increases, so the system response is accelerated.

Remark 4: In the control law, $v_{*}$ represents equivalent control. When neglecting the disturbance term, $v_{*}$ can drive the system states to reach the sliding mode surface, and on which force the system states converge to zero. $v_{1}$ represents the switching control law, which can robustify the system 
against uncertainties and disturbances, and force $s$ converges to zero in finite time with the combined action $v_{*}$.

Remark 5: The closed-loop system will produce chattering due to the non-continuous function $\operatorname{sgn}(s)$. This kind of unexpected high-frequency chattering may arouse system un-modeling dynamics, which will influence the system performance. More seriously, it will cause system instability. In order to reduce chattering, the continuous function of Eq. (36) is used to replace the signal function $\operatorname{sgn}(s)$

$$
\operatorname{sat}(s)=\frac{s}{\|s\|+k}
$$

where $k$ is a very small positive real constant.

\section{Simulation Results}

In order to prove the effectiveness of the proposed approach. Some simulation results for spacecraft in elliptic orbit subject to the bounded external disturbances with $J_{2}$ perturbations or without $J_{2}$ perturbations via the method of a new SMC law are presented. The simulation results are

Table 1. Initial conditions for the chief and deputy satellites.

\begin{tabular}{lll}
\hline Orbit element & Chief & Deputy \\
\hline$a(\mathrm{~km})$ & 7,500 & $7,500.02$ \\
$e$ & 0.1 & 0.0999 \\
$i(\mathrm{deg})$ & 45 & 45.0001 \\
$\Omega(\mathrm{deg})$ & 0 & 0 \\
$\omega(\operatorname{deg})$ & 0 & 0 \\
$f_{0}(\mathrm{deg})$ & 100.05 & 100 \\
\hline
\end{tabular}

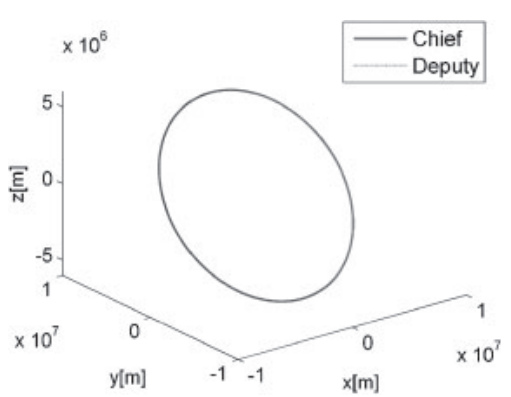

Fig. 2. Relative trajectory with the control of the nonlinear SM function.

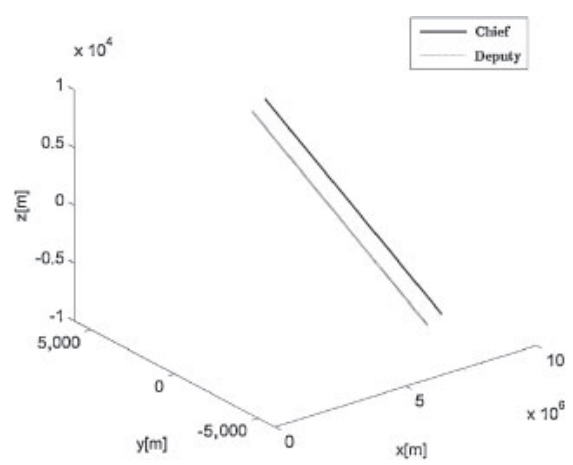

Fig. 3. Detail with enlarged scale of Fig. 2. compared with traditional SMC. The initial conditions of the chief satellite and deputy satellite are shown in Table 1 . The formation vector $l$ is chosen as $l=[1,000 \sin (n t)$ $2,000 \cos (n t) \quad 1,000 \sqrt{3} \sin (n t)] \mathrm{m}$. External disturbances are $\quad\left[\begin{array}{lll}d_{x} & d_{y} & d_{z}\end{array}\right]^{\mathrm{T}}=10^{-3}\left[\begin{array}{lll}-1.2 \sin \dot{f} t & 0.5 \cos 2 \dot{f} t & \sin \dot{f} t\end{array}\right]^{\mathrm{T}}$ $\mathrm{m} / \mathrm{s}^{2}$. The controller parameters are $k_{c}=3, k_{1}=0.8$, $k_{J_{2}}=2, c=3$ and $k=0.5$. The controller parameters can be selected by parameter self-tuning ${ }^{19)}$ or trial and error. In this article the parameters are chosen by trial and error. The simulation results are shown in Figs. 2-13 and Table 2.

Relative trajectory of the chief and deputy with the control of the nonlinear SM function is shown in Fig. 2, from the detail with enlarged scale of Fig. 3, we can see their relative position is kept at $2,000 \sqrt{2} \mathrm{~m}$, which satisfies the requirement we set in this paper.

Figures 4 and 7 describe the time history of tracking errors which are controlled by the SM controller base nonlinear SM function in the presence of $J_{2}$ perturbations and without $J_{2}$ perturbations, respectively. From the figures, one can see the control law proposed in this paper can restrain $J_{2}$ perturbations effectively with good robustness. Figure 9 depicts curves of formation error for traditional SMC with $J_{2}$ perturbations. According to Table 2, we find that the convergence accuracy of tracking error base nonlinear SM function is higher than that for traditional SMC.

The curves of control acceleration with the nonlinear SM
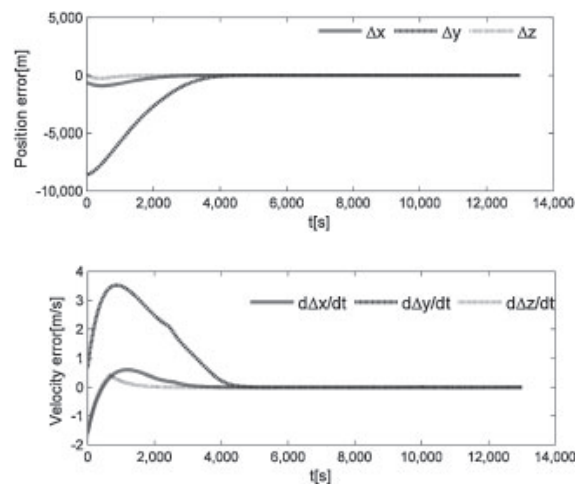

Fig. 4. Tracking error controlled by SMC base nonlinear SM function with consideration of $J_{2}$ perturbations.

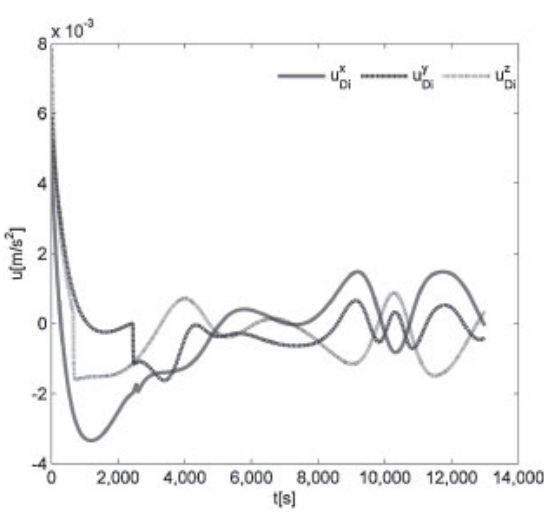

Fig. 5. Control acceleration when controlled by the SMC base nonlinear SM function with consideration of $J_{2}$ perturbations. 
function and traditional SM function are shown in Fig. 5 and Fig. 10, respectively, and Fig. 12 is the comparison of the control acceleration base nonlinear SM function and the traditional SM function in the $y$-axis. It is clear that, at the initial time, the control input base nonlinear function is reduced compared with the traditional SMC. On the whole, according to Table 2, one can conclude the system controller base nonlinear SM function has higher convergence accuracy and lower fuel consumption than that controlled by the traditional SMC.

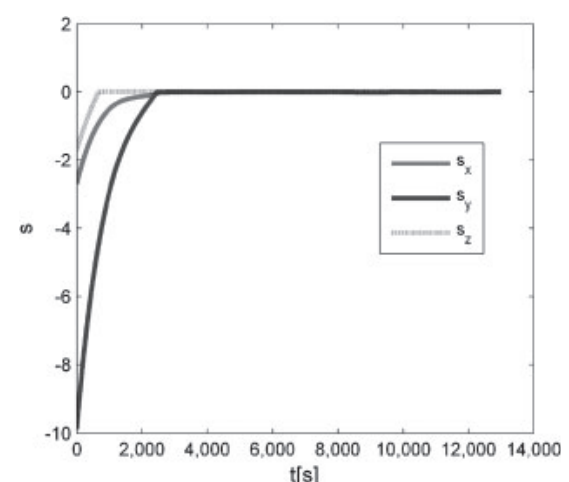

Fig. 6. Time history of the nonlinear sliding mode function with consideration of $J_{2}$ perturbations.
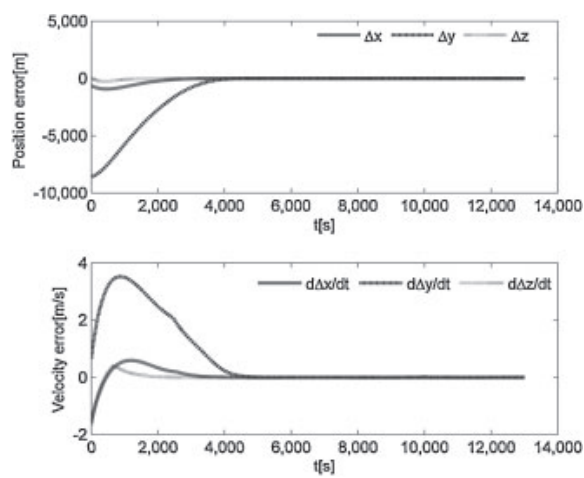

Fig. 7. Tracking error controlled by the SMC base nonlinear SM function without consideration of $J_{2}$ perturbations.

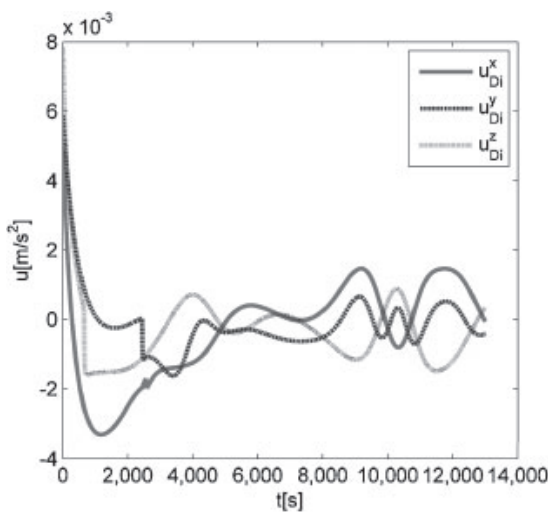

Fig. 8. Control acceleration when controlled by the SMC base nonlinear SM function without consideration of $J_{2}$ perturbations.
Comparing Fig. 6 with Fig. 11, and combining with Fig. 13, the curve of comparison of the sliding mode function between the traditional SMC and SMC base nonlinear $\mathrm{SM}$ function in the $y$-axis, one can see that the initial value of the SM function is decreased, and the time of reaching the sliding surface with the nonlinear SM function is approximately 2,400 s, while it is approximately 3,200 s with the traditional SM function. That verifies the conclusion that the controller proposed in this paper can shorten the reaching phase of SM and also means the system robustness is improved.
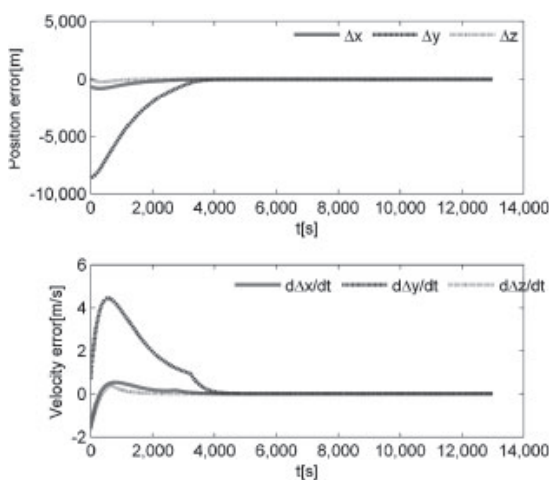

Fig. 9. Tracking error controlled by SMC with consideration of $J_{2}$ perturbations.

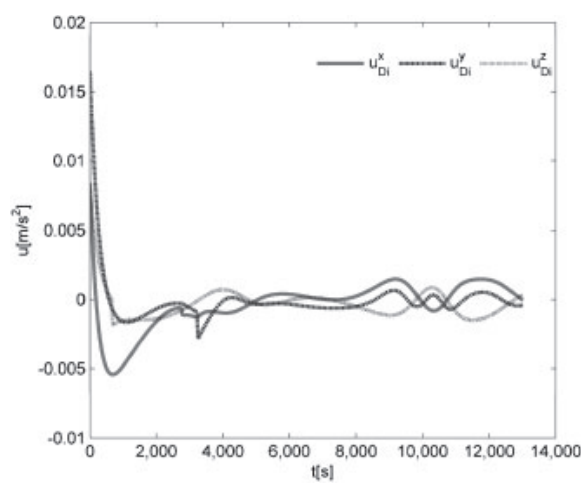

Fig. 10. Control acceleration using SMC with consideration of $J_{2}$ perturbations.

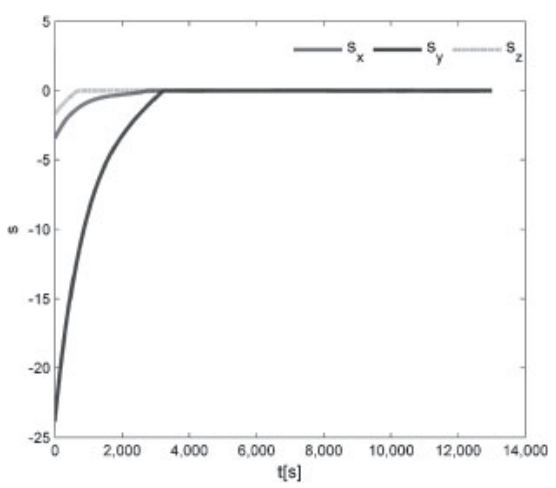

Fig. 11. Time history of the sliding mode function with consideration of $J_{2}$ perturbations. 


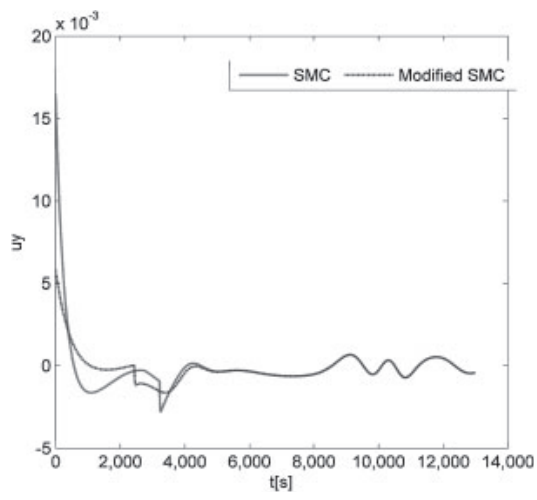

Fig. 12. Comparisons of the control law of the $y$-axis.

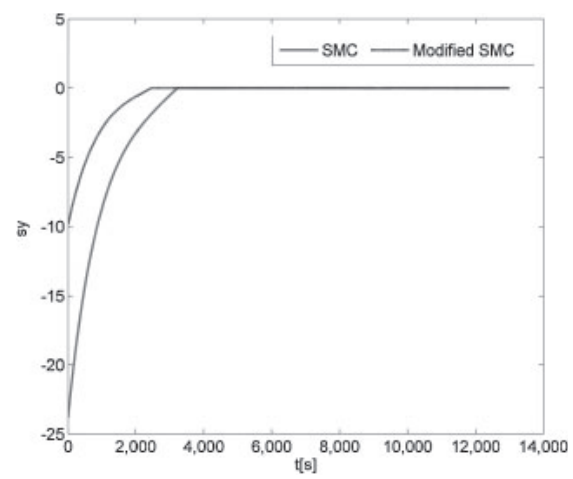

Fig. 13. Comparisons of the sliding mode function of the $y$-axis.

Table 2. Formation errors and fuel consumption.

\begin{tabular}{llccc}
\hline & & $\begin{array}{c}\text { Fuel } \\
\text { consumption } \\
\left(\mathrm{m} / \mathrm{s}^{2}\right)\end{array}$ & $\begin{array}{c}\text { Position } \\
\text { accuracy } \\
(\mathrm{m})\end{array}$ & $\begin{array}{c}\text { Velocity } \\
\text { accuracy } \\
(\mathrm{m} / \mathrm{s})\end{array}$ \\
\hline \multirow{2}{*}{ Modified SMC } & With $J_{2}$ & 33.9770 & 1.19572 & 0.00582 \\
& Without $J_{2}$ & 33.9386 & 1.18768 & 0.00583 \\
\hline SMC & With $J_{2}$ & 36.8483 & 1.19784 & 0.00584 \\
\hline
\end{tabular}

\section{Conclusion}

In this paper, nonlinear differential equations for the formation flying relative motion in elliptic orbit subject to external disturbances in the presence of $J_{2}$ perturbations and without $J_{2}$ perturbations were proposed. For simplifying the relative motion equation base $J_{2}$ perturbations, another imaginary orbit element was introduced. To facilitate the design of control law, both of the SFF models were linearized by feedback linearization. With these models, the formation reconfiguration and keeping controller based on the nonlinear SM function was proposed, which was designed by introducing exponentially proportional to replace the constant gain of the traditional SMC. Simulation results show that the SMC base nonlinear SM function can guarantee high convergence accuracy, low fuel consumption and strong robustness compared to the traditional SMC. This verifies that the proposed control law in this paper is more suitable for practical applications.

\section{Acknowledgments}

The authors acknowledge the financial support from the Major State Basic Research Development Program [grant number 2012CB720000], National Natural Science Foundation of China [grant number 11372034], and Innovative Research Team of Beijing Institute of Technology.

\section{References}

1) Kristiansen, R. and Nicklasson, P. J.: Spacecraft Formation Flying: A Review and New Results on State Feedback Control, Acta Astronaut., $\mathbf{6 5}$ (2009), pp. 1537-1552.

2) Martin, M. and Kilberg, S.: TechSat 21 and Revolutionizing Space Missions using Microsatellites, 15 AIAA/USU Conference on Small Satellites, Logan, UT, 2001, pp. 13-16.

3) Alfriend, K. T.: Dynamics and Control of Earth Orbit Satellite Formations, Lectures in Tinghua University, China, 2010.

4) Hill, G. W.: Researches in Lunar Theory, Am. J. Math., 1 (1878), pp. 5-26.

5) Tschauner, J.: Elliptic Orbit Rendezvous, AIAA J., 5, 6 (1967), pp. 1110-1113.

6) Wong, H., Pan, H., de Queiroz, M. S. and Kapila, V.: Adaptive Learning Control for Spacecraft Formation Flying, Proceedings of 40th IEEE Conference on Decision and Control, Orlando, FL, 2001, pp. 1089-1094.

7) Manikonda, V., Arambel, P. O., Gopinathan, M., Mehra, R. K. and Hadaegh, F. Y.: A Model Predictive Control-Based Approach for Spacecraft Formation Keeping and Attitude Control, Proceedings of the American Control Conference, San Diego, CA, 1999.

8) Xiao, Y. L. and Zhang, X. M.: Orbital Characteristics and Prospective Applications of Cluster of Companion Satellites, Proceedings of the 50th IAF Congress, IAA-99-IAA-11.1.09, Amsterdam, 1999.

9) Yeh, H. H., Nelson, E. and Sparks, A.: Nonlinear Tracking Control for Satellite Formations, J. Guid. Control Dynam, 25, 2 (2002), pp. 376386.

10) Hui, L. and Li, J.: Terminal Sliding Mode Control for Spacecraft Formation Flying, IEEE Trans. Aerospace Electronic Syst., 45, 3 (2009), pp. 835-864.

11) Pongvthithum, R., Veres, S. M. and Gabriel, S. B.: Universal Adaptive Control of Satellite Formation Flying, Int. J. Control, 78, 1 (2005), pp. $45-52$.

12) Xing, G. Q., Parvez, S. A. and Folta, D.: Implementation of Autonomous GPS Guidance and Control for the Spacecraft Formation Flying, American Control Conference, 1999, pp. 4163-4167.

13) Gim, D. W. and Alfriend, K. T.: State Transition Matrix of Relative Motion for the Perturbed Noncircular Reference Orbit, J. Guid. Control Dynam., 26, 6 (2003), pp. 956-971.

14) Battin, R. H.: An Introduction to the Mathematics and Methods of Astrodynamics, AIAA, New York, 1987, pp. 476-504.

15) Meng, Y. H.: Research on Control and Application of Leo Spacecraft Formation Flying, National University of Defense Technology, Changsha, 2006.

16) Khalil, H. K.: Nonlinear Systems, Prentice, New Jersey, 2002.

17) Miller, R. K. and Michel, A. N.: Ordinary Differential Equations, Academic Press, New York, 1982.

18) Carter, T. E. and Humi, M.: Fuel-Optimal Rendezvous near a Point in General Keplerian Orbit, J. Guid. Control Dynam., 10, 6 (1987), pp. 567-573.

19) Cong, B. L., Liu, X. D. and Zhen, C.: Disturbance Observer Based Time-Varying Sliding Mode Control for Uncertain Mechanical System, J. Syst. Eng. Electronics, 23, 1 (2012), pp. 108-118. 